Enterobacteriaceae: comparison of Sensititre, Microscan, Vitek 2, and Etest with broth microdilution. J Clin Microbiol 2017; 55: 2609-16.

5. The European Committee on Antimicrobial Susceptibility Testing. Routine and extended internal quality control for MIC determination and disk diffusion as recommended by EUCAST. Version 11.0. 2021. http:// www.eucast.org

6. Clinical and Laboratory Standards Institute (CLSI). Performance Standards for Antimicrobial Susceptibility Testing. CLSI supplement M100. 30th ed Wayne, PA: Clinical and Laboratory Standards Institute, 2020.

DOI: https://doi.org/10.1016/j.pathol.2021.08.016

\section{First human case of infection with Arsenophonus nasoniae, the male killer insect pathogen}

\section{To the Editor,}

Many arthropod-borne human infections have been recognised in recent years, but this is the first reported case of honeybee-associated Arsenophonus nasoniae bacteraemia.

We present the case of a 37-year-old male who attended the hospital emergency department with sudden onset of fever, rigors, and myalgia 5 hours after sustaining a bee sting to his left thumb that occurred in the evening during April 2018 behind his home garage in Wollongong, New South Wales, Australia. This reaction was unusual for him as he had sustained several bee stings in the past without any severe effects. His other medical problems included eczema, well-controlled with topical corticosteroid cream, and chronic neck pain.

On presentation, he was well-perfused with blood pressure of $137 / 81 \mathrm{mmHg}$ but was tachypnoeic with a respiratory rate of 30 breaths per minute and tachycardic with a heart rate of 145 beats per minute. He was febrile with a temperature of $39.0^{\circ} \mathrm{C}$. Oxygen saturation was $98 \%$ on room air. His left thumb was inflamed but there were no signs of any embedded bee body parts after removal by his wife. The remainder of his physical examination was unremarkable.

Investigations revealed a normal white cell count $10.5 \times 10^{9} / \mathrm{L}$ [reference range (RR) 3.50-11.00] with a mild neutrophilia of $8.29 \times 10^{9} / \mathrm{L}$ (RR $\left.1.7-7.0\right)$. Arterial whole blood showed a normal lactate $1.4 \mathrm{mmol} / \mathrm{L}$ (RR 0.0-2.2). He had normal renal and liver function test results and his urinalysis, electrocardiogram and chest X-ray were all unremarkable. One set of blood cultures was collected into BD BACTEC Plus Aerobic/F and Plus Anaerobic/F culture vials (Becton Dickinson, USA). They were incubated in the BD BACTEC 9000 automated blood culture system. After 4 hours observation and conservative management in the emergency department (ED), he was discharged home. His aerobic blood culture vial signalled positive 48 hours later with Gram-negative bacilli and he was recalled to the ED for further assessment. Clinically, he was doing well with no fever, thus he was not started on any antibiotic and was discharged home again.

The contents of the aerobic vial were subcultured onto horse blood, chocolate and MacConkey agars and these media were incubated at $35^{\circ} \mathrm{C}$. Growth was evident only on horse blood agar and chocolate agar incubated in $5 \% \mathrm{CO}_{2}$ after 48 hours incubation. There was no growth on horse blood agar incubated anaerobically and no growth on
MacConkey agar incubated in ambient air. Colonies were mucoid, grey-white, round, and convex with entire edges. Cells were non-motile, non-spore forming, long rods, occasionally filamentous in young cultures.

The blood culture isolate was further identified by matrixassisted laser desorption ionisation time-of-flight mass spectrometry (MALDI-TOF MS) using a Bruker MALDI Biotyper with Bruker Biotyper 3.4 software and library version V4.0.0.1 (Bruker Daltonik, Germany), according to the manufacturer's instructions, with a spectral score of 1.78 as Arsenophonus nasoniae with good confidence to the genus level.

Confirmation of the species identification was achieved by 16S rRNA PCR and sequencing. DNA extraction was performed from cultures by crude lysate preparation $\left(100^{\circ} \mathrm{C}\right.$ for 10 minutes in molecular grade water). The sequence obtained from the sample was $98.9 \%$ identical to the Arsenophonus nasoniae (ATCC 49151), which was deposited in GenBank (NCBI) under accession NR_042811.1, with eight mismatches in 796 bases sequenced.

Antimicrobial susceptibility testing of the A. nasoniae blood culture isolate was performed using the Vitek 2 semiautomated system (version 08.01; bioMerieux, USA) with the commercially available Vitek 2 AST-N246 card. Antimicrobial susceptibility results are shown in Table 1 .

The patient decided to keep the dead bee in a resealable plastic bag and its remnants were sent to Hawkesbury Institute for the Environment, Western Sydney University for further identification and possible detection of the causative bacterial DNA, 6 weeks after the contact.

The bee was morphologically confirmed as Apis mellifera. Culture was attempted on the bee remnant, but we were not able to identify $A$. nasoniae from the mixed growth. The remnant was homogenised in a TissueLyser (Qiagen, Germany) at $30 \mathrm{~Hz}$ for $4 \mathrm{~min}$ with a $2 \mathrm{~mm}$ sterile steel ball. Total DNA was then extracted using TRIsure (Bioline) following the manufacturer's recommendations. To detect the presence of Arsenophonus sp. DNA in the bee sample, PCR was performed using the Bioline MyTaq Red PCR kit and primer set Ars23S1 (CGTTTGATGAATTCATAGTCAAA)/

Ars23S2 (GGTCCTCCAGTTAGTGTTACCCAAC). ${ }^{2} 200$ ng DNA was used in a $10 \mu \mathrm{L}$ reaction $(2 \mu \mathrm{L}$ DNA, $2 \mu \mathrm{L}$ MyTaq

Table 1 Antimicrobial susceptibility of the Arsenophonus nasoniae blood culture isolate using VITEK 2 Systems Version: 08.01 and AST-N246 card

\begin{tabular}{lc} 
Antimicrobial agent & MIC (mg/L) \\
\hline Ampicillin & 4 \\
Amoxicillin/clavulanic acid & $\leq 2$ \\
Cefazolin & $\leq 4$ \\
Ceftriaxone & $\leq 1$ \\
Ceftazidime & $\leq 1$ \\
Cefepime & $\leq 1$ \\
Meropenem & $\leq 0.25$ \\
Amikacin & 4 \\
Gentamicin & $\leq 1$ \\
Tobramycin & $\leq 1$ \\
Ciprofloxacin & $\leq 0.25$ \\
Norfloxacin & $\leq 0.5$ \\
Nitrofurantoin & $\leq 16$ \\
Trimethoprim & $\leq 0.5$ \\
Trimethoprim/sulfamethoxazole & $\leq 20$ \\
\end{tabular}

MIC, minimal inhibitory concentration. 
red mix, $0.2 \mu \mathrm{L}$ Taq polymerase, $0.4 \mu \mathrm{L} 5$ pmol F primer, 0.4 $\mu \mathrm{L} 5$ pmol R primer, $10 \mu \mathrm{L} \mathrm{H}_{2} \mathrm{O}$ ). Reaction conditions were as follows: initial denaturation $95^{\circ} \mathrm{C}$ for $5 \mathrm{~min}$, then 30 cycles of denaturation for $30 \mathrm{~s}$, annealing at $50^{\circ} \mathrm{C}$ for $30 \mathrm{~s}$ and extension at $72^{\circ} \mathrm{C}$ for $60 \mathrm{~s}$, followed by a final extension $72^{\circ} \mathrm{C}$ for $10 \mathrm{~min}$. Resulting fragments were subjected to Sanger sequencing (in each direction) using the PCR primers. Sequences were then inspected and trimmed in Geneious v10.2.6 (Biomatters, New Zealand) before using a BLASTn search against the NCBI nucleotide database to confirm identity of the fragments. The PCR reaction amplified an approximately 800 bp fragment, which was confirmed using a BLASTn search as showing $97.68 \%$ similarity to A. nasoniae genome (GenBank accession number CP038613.1).

DNA extraction of the human blood culture A. nasoniae isolate was performed using the HiYield Genomic DNA Mini Kit (Real Biotech Company, Taiwan) according to manufacturer's instructions. Nextera library preps for both the human blood culture isolate DNA extract and the honeybee DNA extract followed by whole-genome sequencing using the NovaSeq 6000 Sequencer (Illumina, USA) were also performed according to the manufacturer's instructions. Approximately $22 \mathrm{~Gb}$ of $2 \times 150 \mathrm{nt}$ paired-end reads were generated. Reads were cleaned and trimmed using fastp (https://github.com/OpenGene/fastp) with default parameters. A subsampling of 1000 reads for each sample was taken and blastx search was performed (Diamond BLASTX; https://github.com/bbuchfink/diamond) against NR (release 231) to map the reads to known peptides. The blast output and fasta files were loaded into Megan 6.14.2 (https:// software-ab.informatik.uni-tuebingen.de/download/megan6/ welcome.html) for calculation and visualisation of taxonomic structure.

The metagenomic analysis detected A. nasoniae from the human blood culture DNA extract whereas the honeybee DNA extract contained A. nasoniae, Photorhabdus luminescens, Proteus mirabilis, Haemophilus influenzae but the majority of the reads aligned with the honeybee genome. To establish the relatedness of $A$. nasoniae from the human blood culture DNA extract with the honeybee DNA extract, Snippy (https://github.com/tseemann/snippy v4.6.0) was used to call SNPs based on the public reference genome of A. nasoniae ASM476852v1 (GCA_004768525.1). There were 104,363 SNPs different between the cultured A. nasoniae and the public reference genome. This corresponds to $25 \mathrm{SNPs} / \mathrm{kb}$, congruent with other studies of intraspecies bacterial genomic diversity. Even with $20 \mathrm{~Gb}$ of honeybee metagenomic data (1 read in 9000 mapping to A. nasoniae), coverage of the genome was low at approximately $0.4 \times$ of the $A$. nasoniae genome. Due to the low depth, only 1928 of the 104,363 SNPs could be compared between the honeybee metagenomic sample and the cultured A. nasoniae isolate. However, $95 \%$ of these compared SNPs were identical, demonstrating conclusively that the cultured A. nasoniae from the human blood was the same strain found in the honeybee.

Bee stings causing infection are rare and have not been extensively studied as a medical phenomenon. A range of skin, soft tissue and disseminated infections have been described, including death. ${ }^{3}$ Previously isolated bacteria included Staphylococcus aureus, Streptococcus pyogenes, Pseudomonas aeruginosa, Enterococcus faecalis, Stenotrophomonas maltophilia, and coagulase-negative Staphylococcus species. ${ }^{3}$ There is a case report of co-infection with A. nasoniae and Orientia tsutsugamushi in a traveller to Cambodia, both detected in a skin eschar by qPCR, and with seroconversion to $O$. tsutsugamushi by immunofluorescence and Western blot. The role played by A. nasoniae in the disease pathogenesis was not clear. ${ }^{4}$ The patient was clinically diagnosed with rickettsiosis and recovered following treatment with $200 \mathrm{mg} / \mathrm{day}$ of doxycycline.

Arsenophonus nasoniae is a $\mathrm{\Upsilon}$-proteobacterium from the family Enterobacterales. The genome of A. nasoniae is 3.5 $\mathrm{Mbp}$ in size, a relatively large genome for an insect symbiont. ${ }^{5}$ Arsenophonus nasoniae shows an extensive host range, including arthropods, nematodes, plants, and vertebrates." Arsenophonus nasoniae induces a male-killing phenomenon in the wasp Nasonia vitripennis. ${ }^{6}$ In the honeybee, the presence of $A$. nasoniae is one of several microbial factors that has been suspected in colony collapse disorder, a mysterious condition involving the sudden disappearance of adult worker bees, which may be related to the poor health of the bee. ${ }^{7}$ Thus far A. nasoniae has not been detected in Australian honeybees, but that this may be due to limited reporting and screening in the environment.

The mechanism of A. nasoniae transmission to our patient during the bee sting was unclear, despite genomic analysis which showed close relatedness of our patient $A$. nasoniae DNA to the honeybee $A$. nasoniae DNA. We postulate that the honeybee was in poor health and carried high numbers of A. nasoniae in its hemolymph, on its body, and on its sting organ, possibly due to faecal contamination. Thus, the sting organ contaminated with $A$. nasoniae probably transmitted the bacterium to the patient during the stinging process. The sting organ of the honeybee is composed of a stylet, two barbed lancets, and a venom sac. When the bee utilises its sting, the sting becomes deeply embedded in the skin and quickly advances by alternating the thrusts of both lancets, which, because of the direction of their barbs, can only move forward. Meanwhile, the venom from the sac is injected into the victim. Eventually, the whole stinging mechanism separates from the body of the bee, which kills the bee; however, because of the automatism of the intrinsic muscles, the lancets continue to advance, and the venom is continuously pumped. ${ }^{8}$ In this manner, any bacteria on either the insect's body or its sting, or on the surface of the victim's skin, can be inoculated under the epidermis. Unless meticulously removed, the sting remains in the wound and may facilitate introduction of the infection. Further research is needed to determine the relationship between honeybee stings and infection.

Though our patient presented to the ED septic with detection of $A$. nasoniae in his blood culture, it is yet to be established if this organism releases endotoxin, like other Gram-negative bacteria, triggering an intense inflammatory response during infection. He recovered from this episode without major short-term complication given he was immunocompetent. If this were to occur in an immunosuppressed patient, the outcome may have been more detrimental. 
This case highlights the intricate interactions in nature, between microbes, vectors and humans leading to zoonotic infection. Many other such relationships probably remain to be discovered as part of the One Health movement.

Acknowledgements: The authors thank the patient for his involvement in this study. They also thank Dennis Nelson, Sharon Broadhead, Heather Wren, Dr Sandra Jones and all the NSW Health Pathology staff, Wollongong Hospital, who were involved in cultivation of the Arsenophonus nasoniae; Dr Andrew Ginn and his team at Bacterial Identifications Reference Laboratory (NSW Health Pathology, Westmead, NSW) for technical support and sequencing of the blood culture isolate; Prof Eugene Athan, A/Prof Owen Harris, A/ Prof John Stenos, Yin Peng Lee and Amy-Marie Gilpin for laboratory and technical support.

Conflicts of interest and sources of funding: This study was investigators-initiated, supported by NSW Health Pathology and Hawkesbury Institute for the Environment, Western Sydney University, NSW, Australia. No human genome was sequenced in this study. The authors state that there are no conflicts of interest to disclose.

\section{Kwee Chin Liew ${ }^{1,2}$, Stephen Graves ${ }^{2,3}$, Larry Croft ${ }^{4,5}$, Laura E. Brettell ${ }^{6}$, James Cook ${ }^{6}$, Jeannie Botes ${ }^{7}$, Peter Newton}

${ }^{1}$ NSW Health Pathology Infectious Diseases Department, Wollongong Hospital, NSW, Australia; ${ }^{2}$ Australian Rickettsial Reference Laboratory, University Hospital Geelong, Geelong, Vic, Australia; ${ }^{3}$ NSW Health Pathology Microbiology, Nepean Hospital, Penrith, NSW, Australia; ${ }^{4}$ Deakin Genomic Centre, Deakin University, Vic, Australia; ${ }^{5}$ School of Life and Environmental Sciences, Deakin University, Vic, Australia; ${ }^{6}$ Hawkesbury Institute for the Environment, Western Sydney University, NSW, Australia; ${ }^{7} N S W$ Health Pathology Microbiology, Wollongong Hospital, NSW, Australia

Contact Dr Kwee Chin Liew.

E-mail: lwkwee1@yahoo.com.au

1. Relman DA. Universal bacterial $16 \mathrm{~S}$ rRNA amplification and sequencing In: Persing DH, Smith TF, Tenover FC, White TJ, editors. Diagnostic Molecular Microbiology: Principles and Applications. Washington DC: ASM Press, 1993; 489-95.

2. Thao ML, Baumann P. Evidence for multiple acquisition of Arsenophonus by whitefly species (Sternorrhyncha: aleyrodidae), Curr Microbiol 2004; 48: 140-4.

3. Truskinovsky AM, Dick JD, Hutchins GM. Fatal infection after a bee sting. Clin Infect Dis 2001; 32: e36-8.

4. Edouard S, Subramanian G, Lefevre B, et al. Co-infection with Arsenophonus nasoniae and Orientia tsutsugamushi in a traveller. Vector Borne Zoonotic Dis 2013; 13: 565-71.

5. Darby AC, Choi JH, Wilkes T, et al. Characteristics of the genome of Arsenophonus nasoniae, son-killer bacterium of the wasp Nasonia. Insect Mol Biol 2010; 19 (Suppl 1): 75-89.

6. Ferree PM, Avery A, Azpurua J, Wilkes T, Werren JH. A bacterium targets maternally inherited centrosomes to kill males in Nasonia. Curr Biol 2008; 18: 1409-14.

7. Van Engelsdorp D, Evans JD, Saegerman C, et al. Colony collapse disorder: a descriptive study. PLoS One 2009; 4: e6481.

8. Snodgrass RE. Anatomy and Physiology of the Honeybee. New York: McGraw-Hill, 1925.

DOI: https://doi.org/10.1016/j.pathol.2021.08.011

\section{Acute poisoning by dexmedetomidine-containing chewing gum in a child}

To the Editor,

In this report, we describe an unusual case of acute poisoning caused by dexmedetomidine-adulterated chewing gum.

A 7-year-old boy was admitted to the Department of Pediatrics and Adolescent Medicine with loss of consciousness The patient became unarousable shortly after consuming a piece of chewing gum in ordinary packaging. The chewing gum was retrieved from the patient's mouth and he was immediately sent to the hospital. On arrival to the Accident and Emergency department, his blood pressure was 119/76 $\mathrm{mmHg}$, with pulse rate of 76 beats per minute and respiratory rate of 22 breaths per minute. He was unresponsive with a Glasgow Coma Score of 4/15 (E1V2M1). Pinpoint pupils were observed, so opioid overdose was initially suspected and naloxone was given. Initial laboratory investigations, including arterial blood gas, complete blood count, clotting profile, liver and renal functions, electrolytes, blood glucose and ammonia were all unremarkable. Ethanol level in the blood was undetectable. Computed tomography of the brain did not reveal any focal lesion or abnormality. The patient was admitted to the paediatrics intensive care unit (PICU) for close monitoring and endotracheal intubation was performed for airway protection. He gradually regained consciousness and was extubated on the following day. He recovered uneventfully and was discharged a week after admission. Further follow-up physical examination and brain imaging at 2 months after discharge showed absence of long-term sequelae.

Toxicology investigation was performed on the chewing gum remains and patient's urine specimen. The chewing gum was extracted by liquid-liquid extraction as follows: the specimen was first extracted with $70 \%$ acetonitrile $(0.1 \mathrm{~g} / \mathrm{mL})$ and sonicated for $30 \mathrm{~min}$. Supernatant was collected after centrifugation; $0.5 \mathrm{~mL}$ of the supernatant was mixed with 3.5 $\mathrm{mL}$ purified water and $50 \mu \mathrm{L}$ of pinazepam (internal standard). The mixture was then extracted using De-tox Tube A by mechanical shaking for $30 \mathrm{~min}$. After centrifugation, 1.25 $\mathrm{mL}$ of the upper organic phase was aspirated, dried under compressed air, and reconstituted in $0.8 \mathrm{~mL} 30 \%$ acetonitrile. The supernatant was injected onto the high performance liquid chromatograph (HPLC) followed by diode array detection (DAD). The same extract was also subjected to a separate tandem mass spectrometry (MS/MS) analysis for confirmation. The urine specimen was analysed by LC-MS/ MS after 1:5 dilution with water. Dexmedetomidine was detected in both the chewing gum remains and the patient's urine specimen. Other drugs detected in the urine, including midazolam, naloxone and rocuronium, were all given after hospitalisation.

Dexmedetomidine is an alpha-2 adrenergic receptor agonist with sedative, anxiolytic, analgesic and cardiovascular stabilising effects. It is notable for its ability to achieve sedation without significant respiratory depression. Dexmedetomidine was developed in the 1980s and approved by the Food and Drug Administration for human use in 1999. It has a distribution half-life of 6 minutes and plasma half-life of 2 hours. ${ }^{1}$ Common uses include sedation in intensive care and for paediatric procedures, controlled 